7th International Workshop on Astronomy and

Relativistic Astrophysics (IWARA 2016)

International Journal of Modern Physics: Conference Series

Vol. 45 (2017) 1760038 (6 pages)

(C) The Author(s)

DOI: $10.1142 / \mathrm{S} 2010194517600382$

\title{
Bayesian Inference Applied to Pulsar's Models
}

\author{
Rubens M. Marinho Jr., Heitor O. de Oliveira \\ Departamento de Física \\ Instituto Tecnológico de Aeronáutica (ITA) \\ 12228-900 São José dos Campos, São Paulo, Brazil \\ marinho@ita.br,heitor.de.oliveira@hotmail.com \\ Nadja S. Magalhães, Rodolfo Valentim \\ Departamento de Física \\ Universidade Federal de São Paulo (UNIFESP) \\ 09913-030 Diadema, São Paulo, Brazil \\ nadjasm@gmail.com,r.valentim@gmail.com \\ Jaziel G. Coelho \\ Divisão de Astrofíisica \\ Instituto Nacional de Pesquisas Espaciais (INPE) \\ 12227-010 São José dos Campos, São Paulo, Brazil \\ jaziel.coelho@inpe.br \\ Márcio E. S. Alves \\ Instituto de Ciência e Tecnologia \\ Universidade Estadual Paulista (UNESP) \\ 12247-016 São José dos Campos, São Paulo, Brazil \\ marcio.alves@ict.unesp.br
}

Published 15 August 2017

\begin{abstract}
The goal of this work is to apply Bayesian statistics to the problem of pulsars in order to compute the Bayes factor and investigate which one among different EoS could better fit known pulsar data, regarding the rate of decrease of the angular velocity versus the angular velocity itself. We also find the posterior distribution and the best fit for some relevant parameters of the pulsar like the mass and the magnetic field.
\end{abstract}

Keywords: Pulsar, Equation of State, Bayesian Statistics.

PACS numbers: 04.30.-w, 04.30

This is an Open Access article published by World Scientific Publishing Company. It is distributed under the terms of the Creative Commons Attribution 4.0 (CC-BY) License. Further distribution of this work is permitted, provided the original work is properly cited. 


\section{Introduction}

There are strong evidences that pulsars are rotating magnetized neutron stars emitting electromagnetic radiation in a wide range of wavelengths. The decay of a pulsar's rotation angular frequency is an observational evidence that the rotation energy decreases with time. However, it is well known that the canonical model for magnetic dipole radiation cannot precisely explain the energy loss. There is a variety of models trying to explain the phenomenon, such as the energy loss due to the emission of gravitational radiation ${ }^{1}$, quantum vacuum friction ${ }^{2}$ and interaction of the magnetic field with the plasma surrounding the star, and/or also variations in magnetic field, angle inclination ${ }^{3-5}$, moment of inertia, etc. Moreover, the equations that describe the phenomenon are functions of the mass-radius relation of the neutron star and so far it is not known which equation of state (EoS) for nuclear matter should be used to describe the star's interior.

In the canonical model, pulsars are described as spheres which have a rotating magnetic dipole and with the magnetic dipole axis misaligned with respect to its axis of rotation by which the star radiates its rotational energy as electromagnetic energy. In such model, it does not consider the variation of the moment of inertia $(\dot{I}=0)$.

\section{Equations of State for Neutron Stars}

We selected up-to-date nuclear equations of state (EoS) for this study. In particular, we adopt the parametrizations GM1, TM1, and NL3 (see Refs. ${ }^{6,7}$ ). Following the Hartle's formalism, we compute rotating configurations, accurate up to second-order

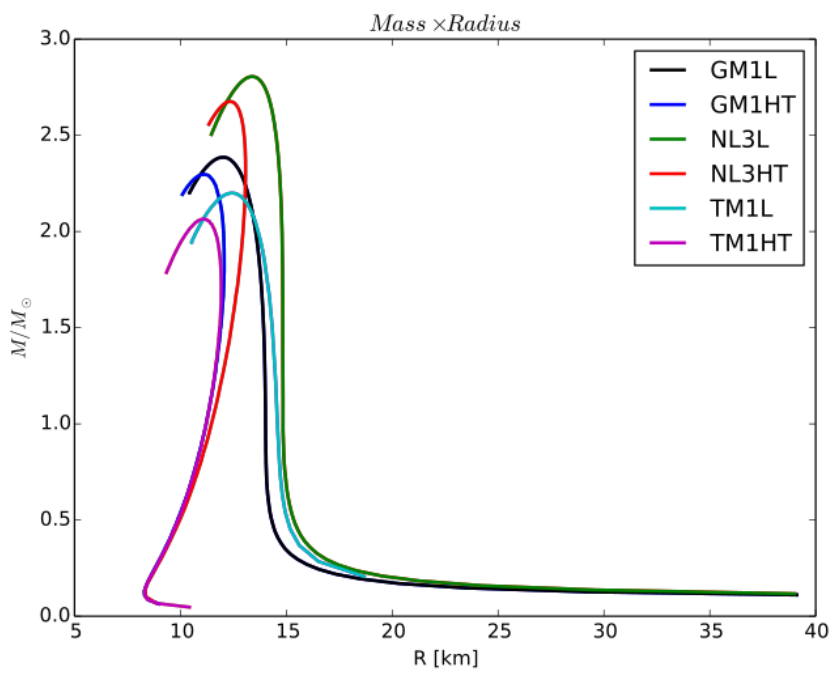

Fig. 1. Mass-Radius relations for different equations of state for neutron stars. 
in $\Omega$, with the same central density as the seed static, non-rotating configurations. The mass-radius relations for non-rotating configurations in the case of local charge neutrality are shown in Figure 1.

\section{Bayes' Theorem Probing EoS}

In the following we consider Bayes' theorem probing EoS ${ }^{8}$. We are considering the simple canonical model for pulsars, for which ${ }^{6}$

$$
\dot{\Omega}=-\frac{5 \Omega^{3} B^{2} R^{4}}{12 c^{3} M},
$$

where $\Omega$ is the angular frequency of arrival of the pulses, $\dot{\Omega}$ is its time derivative, $M$ is the mass of the pulsar, $R$ is its radius and $B$ is the magnetic field at the pole of the star.

For each EoS Figure 1 shows a different function $R=R(M)$. Therefore, since $\Omega$ and $\dot{\Omega}$ are observational data it is possible to estimate the distributions of $M$ and $B$ for pulsars.

We denote the data by $D=\left\{d_{1}, d_{2}, \cdots, d_{N}\right\}$ for $N$ pulsars. We consider

$$
d_{i}=\dot{\Omega}_{i}+e_{i}, \quad i=1,2, \cdots, N
$$

where $e_{i}$ describes spurious information that we name "noise".

The Bayes' theorem states that given the hypothesis $H_{k}$ that the true EoS is $k$, then the posterior probability density function (PDF) for the parameter $\theta$ is

$$
P\left(\theta \mid H_{k}, D\right)=\frac{P\left(D \mid H_{k}, \theta\right) P\left(\theta \mid H_{k}\right)}{P\left(D \mid H_{k}\right)}
$$

where, $P\left(D \mid H_{k}, \theta\right)$ is the likelihood function, $P\left(\theta \mid, H_{k}\right)$ is the prior's distribution and $P\left(D \mid H_{k}\right)$ is a normalization constant.

It is reasonable to assume a Gaussian likelihood function

$$
P\left(D \mid H_{k}, \theta\right)=\frac{1}{(\sqrt{2 \pi})^{N} \prod_{i} \sigma_{i}} \exp \left[-\frac{1}{2} \sum_{i=1}^{N} \frac{\left(d_{i}-\dot{\Omega}_{i}\right)^{2}}{\sigma_{i}^{2}}\right],
$$

where $\sigma_{i}$ is the standard deviation for each measurement.

\section{Bayesian Evidence}

Bayesian Evidence (BE) derives from Bayes' theorem and allows the comparison of different models by marginalizing the likelihood function 4. Integrating over all the parameters space

$$
P\left(D \mid H_{i}\right)=\int P\left(D \mid H_{i}, \theta\right) P(\theta) d \theta
$$




\section{R. M. Marinho Jr. et al.}

BE is based on Occham's razor principle and its test of different models is performed through the Bayes factor

$$
B_{i j}=\frac{P\left(D \mid H_{i}\right)}{P\left(D \mid H_{j}\right)} .
$$

In this case, $E=B_{i j}$ and the interpretation of BE is shown in Table 1 (see Ref. ${ }^{9}$ ).

Table 1. Bayesian Evidence.

\begin{tabular}{cc}
\hline \hline & Support \\
\hline $0<2 \ln E \leq 2$ & Not worth more than a bare mention \\
$2 \leq 2 \ln E \leq 6$ & Significant/Weak \\
$6 \leq 2 \ln E \leq 10$ & Strong to very strong/Significant \\
$10<2 \ln E$ & Decisive/Strong \\
\hline \hline
\end{tabular}

\section{Results}

By considering the data of the 9 pulsars with known braking index: B0531+21 (Crab), B0540-69, B0833-45 (Vela), B1509-58, J1846-0258, J1833-1034, J1119-6127 and J1734-3333, we have evaluated the posterior PDF for the magnetic field $B$ of the pulsar. Examples of results with two EoS are shown in Figures 3 and 4 . Note that the magnetic field distribution and its mean value vary with the chosen EoS.

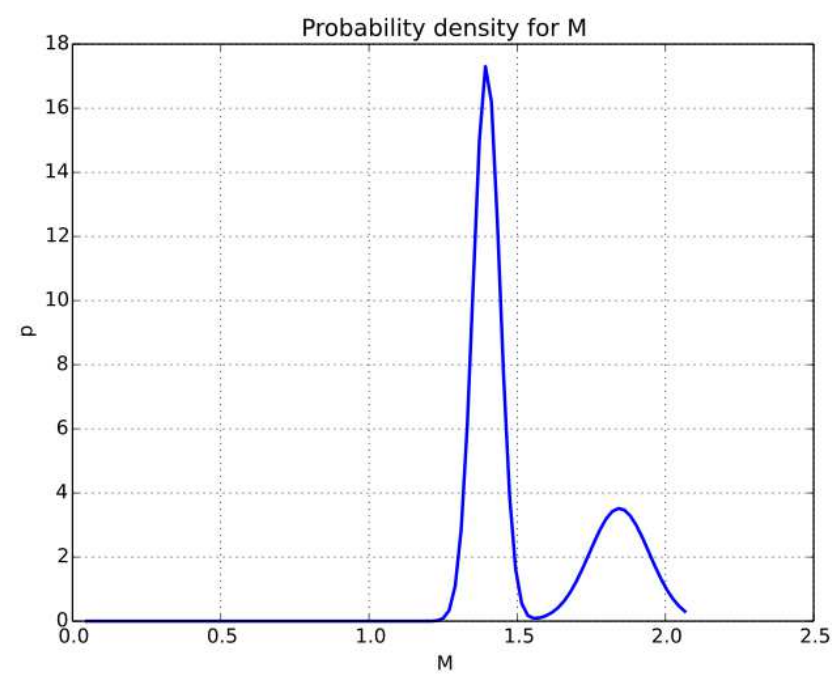

Fig. 2. Posterior PDF for the mass of the pulsars (in units of solar masses). The posterior is very similar to the bimodal prior distribution that was used used. 


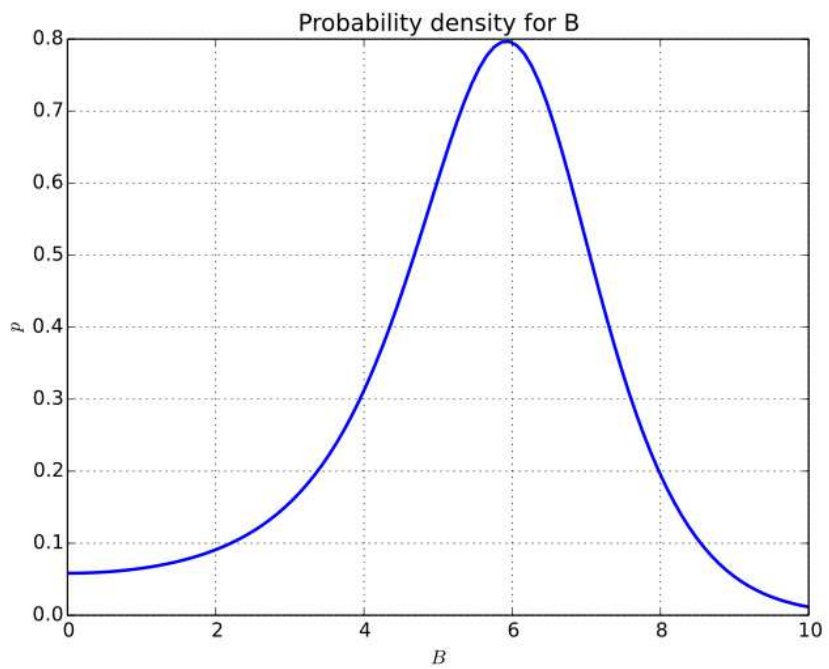

Fig. 3. The posterior PDF for the magnetic field of the neutron star obtained by considering the parametrization TM1HT.

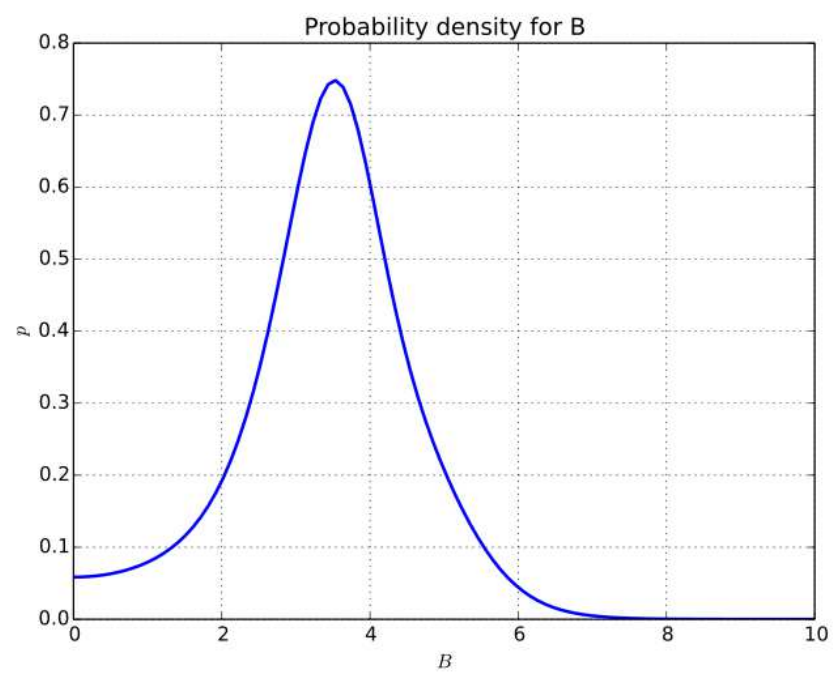

Fig. 4. The posterior PDF for the magnetic field of the neutron star obtained by considering the parametrization TM1L. Notice that different EoS lead to different estimates for the magnetic field.

On the other hand, the posterior PDF for the mass distribution of pulsars ${ }^{10,11}$ has a weak dependence on the EoS, as Figure 2 illustrates. Furthermore, this posterior is very similar to the prior mass distribution, indicating that the former is not significantly affected by the choice of the EoS. 
Table 2. The BE for the EoS we are considering. The ratios are calculated by using the models in the first column in the numerator and the models in the first line in the denominator.

\begin{tabular}{|l|c|c|c|c|c|c|}
\hline & GM1L & GM1HT & NL3L & NL3HT & TM1L & TM1HT \\
\hline GM1L & 0 & -0.16 & 0.93 & 0.78 & -0.18 & 0.65 \\
\hline GM1HT & 0.16 & 0 & 1.09 & 0.94 & -0.02 & 0.81 \\
\hline NL3L & -0.93 & -1.09 & 0 & -0.15 & -1.11 & -0.28 \\
\hline NL3HT & -0.78 & -0.94 & 0.15 & 0 & -0.96 & -0.13 \\
\hline TM1L & 0.18 & 0.02 & 1.11 & 0.96 & 0 & 0.83 \\
\hline TM1HT & -0.65 & -0.81 & 0.28 & 0.13 & -0.83 & 0 \\
\hline
\end{tabular}

Finally, by marginalizing over $M$ and $B$ we find the BE for all the pairs of EoS considered in this work. As can be seen in the Table 2, none of the models are strongly favored when compared with the others. This can be explained in one hand by the few data considered and, on the other hand, by the similar predictions of the different models regarding the mass-radius relation.

\section{Acknowledgments}

The authors acknowledge FAPESP for support under the thematic project \# 2013/26258-4. J. G. C. acknowledges the support of FAPESP N. 2013/15088-0. H. O. O. thanks Coordenação de Aperfeiçoamento de Pessoal de Nível Superior (CAPES) for the financial support.

\section{References}

1. J. C. N. de Araujo, J. G. Coelho, and C. A. Costa, JCAP 7, 023 (2016).

2. J. G. Coelho, J. P. Pereira, and J. C. N. de Araujo, ApJ, 823, 97 (2016).

3. J. C. N. de Araujo, J. G. Coelho, and C. A. Costa, European Physical Journal C 76, $481(2016)$.

4. J. C. N. de Araujo, J. G. Coelho, and C. A. Costa, ApJ 831, 35 (2016).

5. J. C. N. de Araujo, J. G. Coelho, and C. A. Costa, arXiv:1610.10092 (2016).

6. N. K. Glendenning, Compact Stars: nuclear physics, particle physics, and general relativity (Springer-Verlag New York, USA 2000).

7. J. G. Coelho et al, $A \mathscr{G} A$ 599, A87 (2017).

8. G. L. Bretthorst, Bayesian Spectrum Analysis and Parameter Estimation; Lecture Notes in Statistics, 48 (Springer-Verlag, New York, USA, 1988).

9. R. Valentim, E. Rangel and J. E. Horvath, MNRAS 414, 1427 (2011).

10. J. E. Horvath and R. Valentim, arXiv:1607.06981 (2016).

11. R. E. Kass and A. E. Raftery, Journal of the American Statistical Association 90, 773 (1995). 